\title{
New Exponential Stability Criteria for Certain Neutral Integro-Differential Equations
}

\author{
Melek Gözen \\ Erciş Management Faculty,Van Yüzüncü Y1l University 65080, Van - Turkey
}

Geliş / Received: 25/10/2019, Kabul / Accepted: 21/02/2020

\begin{abstract}
In this work, the exponential stability of zero solution of some neutral integro-differential equations of the first order (NIDE) with discrete and distributed time-varying delays is discussed. A new result that has sufficient conditions on the exponential stability of zero solution is proved by aid a new Lyapunov-Krasovskii functional, the Leibniz-Newton formula and a matrix inequality. The result of this paper extends and improves some former results on the topic in the literature.
\end{abstract}

Keywords: Neutral differential equation, first order, exponential stability, time-varying delay, the direct method of Lyapunov

\section{Bazı Neutral İntegro- Diferansiyel Denklemler için Yeni Üstel Kararlıık Kriterleri}

\section{Özet}

$\mathrm{Bu}$ çalışmada, birinci basamaktan ayrık ve değişken gecikmeli neutral integro- diferansiyel denklemlerin (NIDE) sıfir çözümünün üstel kararlılığı incelenmektedir. Uygun bir Lyapunov-Krasovski fonksiyeli, LeibnizNewton formülü ve matris eşitsizliği yardımıyla ele alınan denklemin sıfir çözümünün üstel kararlılığı için yeter şartlar içeren yeni bir sonuç ispatlanmaktadır. Bu çalışma literatürdeki konuyla ilgili önceki sonuçları genişletmekte ve geliştirmektedir.

Anahtar Kelimeler: Neutral diferansiyel denklem, birinci mertebe, üstel kararlılık, değişken gecikme, Lyapunov 'un doğrudan metodu

\section{Introduction}

It is known that neutral differential equations are retarded systems that often appear in many various scientific areas such as physics, biology, chemistry, biophysics, mechanics, aerodynamics, economy, atomic energy, control theory, information theory, population dynamics, electrodynamics of complex media and so on. Therefore, qualitative behaviors of solutions NIDEs, stability, boundedness, convergence, instability, integrability, globally existence of solutions, etc., have been extensively investigated in the literature by this time. For a comprehensive treatment of these qualitative properties of solutions of NIDEs and some applications, we refer the readers to the papers or books of Hale et al (1993), Boyd et al (1994), Agarwal and Grace (2000), ElMorshedy and Gopalsamy (2000), Fridman (2001), Fridman (2002), Gu et al (2003), Park (2004), Kwon and Park (2006), Sun and Wang (2006), Kwon and Park (2008), Park and Kwon (2008), Deng et al (2009), Liao et al (2009), Li 
(2009), Nam and Phat (2009), Rojsiraphisal and Niamsup (2010), Chen et al (2011), Chen and Huabin (2012), Tunç and Altun (2012), Tunç (2013), Pinjai and Mukdasai (2013), Li and Fu (2013), Keadnarmol et al (2014), Chatbupapan et al (2016), Gözen and Tunç (2017a and b), Tunç and Mohammed (2017), Gözen and Tunç (2018), Tunç and Tunç (2018), Hristova and Tunç (2019), Slyn'ko and Tunç (2019) and the references can be found in these sources.

In a particular case, we should mention the following related paper on the qualitative behaviors of the solutions of NIDEs .

Chatbupapan et al (2006) considered a scalar NIDE with mixed interval time varying delays:

$$
\begin{aligned}
& \frac{d}{d t}[x(t)+p x(t-\tau(t))]=-a x(t)+b \tanh x(t-\sigma(t)) \\
& +c \int_{t-\rho(t)}^{t} x(s) d s, t \geq 0
\end{aligned}
$$

Chatbupapan et al. (2006) discussed the delaydependent exponential stability of solutions of NIDE (1.1). Based on a class of LyapunovKrasovskii functionals, a model transformation, the decomposition technique of constant coefficients, the Leibniz-Newton formula and linear matrix inequality (LMI), sufficient conditions are established to guarantee the exponentially stability of the zero of NIDE (1.1) by Chatbupapan et al. (2006) In addition, in this paper a few numerical examples are given to illustrate the effectiveness and applicability of the established conditions.

Motivated by the results of Chatbupapan et al. (2006) and the above mentioned works, we consider the following scalar NIDE with variable delays of the form

$$
\begin{aligned}
\frac{d}{d t}[x(t)+p x(t-\tau(t))] \\
=-h(x)+b(t) \tanh x(t-\sigma(t)) \\
\quad+\int_{t-\rho(t)}^{t} C(t, s) x(s) d s,
\end{aligned}
$$

where $t \in R^{+}, R^{+}=[0, \infty), b(t) \quad$ is positive continuous function, $C(t, s)$ is a continuous function for $0 \leq s \leq t<\infty$ such that $|C(t, s)|<1, \quad p$ is a real constant with $|p|<1$, $\tau(t), \quad \sigma(t)$ and $\rho(t)$ are continuously differentiable functions such that

$0 \leq \tau_{1} \leq \tau(t) \leq \tau_{2}, \quad \dot{\tau}(t) \leq \tau_{d}<\infty$

$0 \leq \sigma_{1} \leq \sigma(t) \leq \sigma_{2}, \quad \dot{\sigma}(t) \leq \sigma_{d}<\infty$

$0 \leq \rho_{1} \leq \rho(t) \leq \rho_{2}$,

where $\tau_{1}, \tau_{2}, \sigma_{1}, \sigma_{2}, \rho_{1}, \rho_{2}, \tau_{d}$ and $\sigma_{d}$ are given positive real constants.

Let

$$
h_{1}(x)= \begin{cases}\frac{h(x)}{x}, & x \neq 0 \\ h^{\prime}(0), & x=0\end{cases}
$$

such that

$$
h^{\prime}(x) \geq 1, x \neq 0 .
$$

For each solution $x(t)$ of (1.2), we assume the initial condition

$$
x_{0}(t)=\phi(t), t \in[-w, 0]
$$

where $\quad \phi \in C([-w, 0], R) \quad$ and $w=\max \left\{\tau_{2}, \sigma_{2}, \rho_{2}\right\}$.

In this paper, we prove an exponential stability theorem, which included sufficient conditions, such that the zero solution of NIDE (1.2) is exponential stability. To prove that theorem we benefit from the Lyapunov second method. For this define a new Lyapunov functional such that it is positive definite and its derivative along the solutions of NIDE (1.2) is negative definite. By this work, our aim is extend and improve some related results in the literature.

Definition 1.1 (Kwon and Park (2006). The zero solution of equation (1.2) is exponentially stable, if there exist positive real constants $\alpha, \beta$ such that, for each $\phi(t) \in C([-w, 0], R)$, the solution $x(t, \phi)$ of equation NIDE (1.2) satisfies

$$
\|x(t, \phi)\| \leq \beta\|\phi\| e^{-a t}, t \geq 0 .
$$


Lemma 1.1 (Gu et al. (2003), (Jensen's inequality)). For any symmetric positive definite matrix $Q$, positive real number $h$ and vector function $\dot{x}(t):[-h, 0] \rightarrow R^{n}$ the following integral is well defined

$$
\begin{aligned}
& -h \int_{-h}^{0} \dot{x}^{T}(s+t) Q \dot{x}(s+t) d s \\
& \leq-\left(\int_{-h}^{0} \dot{x}(s+t) d s\right)^{T} Q\left(\int_{-h}^{0} \dot{x}(s+t) d s\right) .
\end{aligned}
$$

Lemma 1.2 (Gu et al. (2003)). For any constant symmetric positive definite matrix $Q \in R^{n \times n}, h(t)$ a discrete time-varying delay with $0<h_{1} \leq h(t) \leq h_{2}$, the vector function $w:\left[-h_{2}, 0\right] \rightarrow R^{n}$ such that the integrations concerned are well defined, we have

$$
\begin{gathered}
-\left[h_{2}-h_{1}\right] \int_{-h_{2}}^{-h_{1}} w^{T}(s) Q w(s) d s \\
\leq-\int_{-h(t)}^{-h_{1}} w^{T}(s) d s Q \int_{-h(t)}^{-h_{1}} w(s) d s \\
\quad-\int_{-h_{2}}^{-h(t)} w^{T}(s) d s Q \int_{-h_{2}}^{-h(t)} w(s) d s .
\end{gathered}
$$

where $h_{1}, h_{2} \in R$.

We now investigate the exponential stability of zero solution of NIDE (1.2). It is clear that

$$
0=x(t)-x(t-\tau(t))-\int_{t-\tau(t)}^{t} \dot{x}(s) d s
$$

and

$$
0=x(t)-x(t-\gamma \tau(t))-\int_{t-\gamma \tau(t)}^{t} \dot{x}(s) d s,
$$

where $\gamma$ is a given positive real constant. Then, we have

$$
0=r_{1} x(t)-r_{1} x(t-\tau(t))-r_{1} \int_{t-\tau(t)}^{t} \dot{x}(s) d s
$$

and

$$
0=r_{2} x(t)-r_{2} x(t-\gamma \tau(t))-r_{2} \int_{t-\gamma \tau(t)}^{t} \dot{x}(s) d s
$$

where $r_{1}, r_{2} \in R$ will be chosen later. By equalities (1.3)-(1.6), NIDE (1.2) can be written as the following form

$$
\begin{aligned}
& \frac{d}{d t}\left[p_{1} x(t)+p_{2} x(t-\tau(t))+x(t-\gamma \tau(t))\right. \\
+ & \left.\int_{t-\gamma \tau(t)}^{t} \dot{x}(s) d s-p_{1} \int_{t-\tau(t)}^{t} \dot{x}(s) d s\right] \\
= & -\left(a_{1}-r_{1}-r_{2}\right) h(x(t))-\left(a_{2}+r_{1}\right) h(x(t-\tau(t)) \\
& -\left(a_{2}+r_{1}\right) \int_{t-\tau(t)}^{t}(h(x(s)))^{\prime} d s-r_{2} h(x(t-\gamma \tau(t))) \\
& -r_{2} \int_{t-\gamma \tau(t)}^{t}(h(x(s)))^{\prime} d s+b(t) \tanh x(t-\sigma(t)) \\
& +\int_{t-p(t)}^{t} C(t, s) x(s) d s .
\end{aligned}
$$

Let

$$
\begin{gathered}
D(t)=p_{1} x(t)+p_{2} x(t-\tau(t))+x(t-\gamma \tau(t)) \\
\left.+\int_{t-\gamma \tau(t)}^{t} \dot{x}(s) d s-p_{1} \int_{t-\tau(t)}^{t} \dot{x}(s) d s\right] .
\end{gathered}
$$

Hence, it follows from (1.7) that

$$
\begin{aligned}
\dot{D}(t) & =-\left(a_{1}-r_{1}-r_{2}\right) h(x(t))-\left(a_{2}+r_{1}\right) h(x(t-\tau(t)) \\
- & \left.\left(a_{2}+r_{1}\right) \int_{t-\tau(t)}^{t}(h(x(s)))^{\prime} d s\right] \\
& -r_{2} h(x(t-\gamma \tau(t))) \\
& -r_{2} \int_{t-\gamma \tau(t)}^{t}(h(x(s)))^{\prime} d s+b(t) \tanh x(t-\sigma(t)) \\
& +\int_{t-p(t)}^{t} C(t, s) x(s) d s .
\end{aligned}
$$

\section{Material and Method}

We first present some notations, which are needed later.

Let

$\Sigma=\left[\Omega_{(i, j)}\right]_{25 \times 25,}(i, j=1,2, \ldots, 25)$,

where $\Omega$ is a symmetric matrix, that is,

$\Omega_{(i, j)}=\Omega_{(j, i)}$,

and 


$$
\begin{aligned}
& \Omega_{(1,1)}=2 k_{1} \alpha-2 q_{1} \\
& \Omega_{(4,14)}=\Omega_{(4,15)}=\Omega_{(4,16)}=0 \text {, } \\
& \Omega_{(1,2)}=q_{1} p_{1}-q_{2}+k_{1}\left(r_{1}+r_{2}-a_{1}\right) h(x(t)), \quad \Omega_{(4,17)}=\Omega_{(4,18)}=\Omega_{(4,19)}=0 \text {, } \\
& \Omega_{(1,3)}=q_{1} p_{2}-q_{3}-k_{1}\left(r_{1}+a_{2}\right) h(x(t-\tau(t))), \quad \Omega_{(4,20)}=\Omega_{(4,21)}=\Omega_{(4,22)}=0 \text {, } \\
& \Omega_{(1,4)}=-q_{1} p_{1}-q_{4}-k_{1}\left(r_{1}+a_{2}\right) \text {, } \\
& \Omega_{(1,5)}=q_{1}-q_{5}-k_{1} r_{2} h_{1}(x(t-\gamma \tau(t))) \text {, } \\
& \Omega_{(1,6)}=q_{1}-q_{6}-k_{1} r_{2} \text {, } \\
& \Omega_{(1,7)}=\Omega_{(1,8)}=\Omega_{(1,9)}=\Omega_{(1,10)}=0 \text {, } \\
& \Omega_{(1,11)}=k_{1} b(t), \Omega_{(1,12)}=\Omega_{(1,13)}=\Omega_{(1,14)}=0 \text {, } \\
& \Omega_{(1,15)}=\Omega_{(1,16)}=\Omega_{(1,17)}=\Omega_{(1,18)}=0 \text {, } \\
& \Omega_{(1,19)}=\Omega_{(1,20)}=\Omega_{(1,21)}=0 \text {, } \\
& \Omega_{(1,22)}=\Omega_{(1,23)}=\Omega_{(1,24)}=0, \Omega_{(1,25)}=k_{1} \text {, } \\
& \Omega_{(2,2)}=2 q_{2} p_{1}+k_{2}+k_{3}+k_{4}+k_{5}+k_{6} \tau_{2}^{2} \text {, } \\
& +k_{7} \gamma^{2} \tau_{2}^{2}+k_{8}+k_{9} \sigma_{2}^{2} \\
& +w_{5} \tau_{1}^{2}+w_{6} \gamma^{2} \tau_{1}^{2}+w_{7}\left(\tau_{2}-\tau_{1}\right)^{2} \\
& +w_{8} \gamma^{2}\left(\tau_{2}-\tau_{1}\right)^{2} \\
& +w_{10}\left(\sigma_{2}-\sigma_{1}\right)^{2}+k_{10}+w p_{2}^{2}+w_{9} \sigma_{1}^{2}, \\
& \Omega_{(2,3)}=q_{2} p_{2}+q_{3} p_{1}, \Omega_{(2,4)}=-q_{2} p_{1}+q_{4} p_{1} \text {, } \\
& \Omega_{(2,5)}=q_{2}+q_{5} p_{1}, \Omega_{(2,6)}=q_{2}+q_{6} p_{1} \text {, } \\
& \Omega_{(2,7)}=\Omega_{(2,8)}=\Omega_{(2,9)}=0 \text {, } \\
& \Omega_{(2,10)}=\Omega_{(2,11)}=\Omega_{(2,12)}=0, \Omega_{(2,13)}=-q_{7} a_{1} h_{1}(x(t)) \text {, } \\
& \Omega_{(2,14)}=\Omega_{(2,15)}=\Omega_{(2,16)}=0 \text {, } \\
& \Omega_{(2,17)}=\Omega_{(2,18)}=\Omega_{(2,19)}=0 \text {, } \\
& \Omega_{(2,20)}=\Omega_{(2,21)}=\Omega_{(2,22)}=0 \text {, } \\
& \Omega_{(2,23)}=\Omega_{(2,24)}=\Omega_{(2,25)}=0 \text {, } \\
& \Omega_{(3,3)}=2 q_{3} p_{2}-k_{3} e^{-2 \alpha \tau_{2}}+k_{3} \tau_{d}, \\
& \Omega_{(3,4)}=-q_{3} p_{1}+q_{4} p_{2}, \Omega_{(3,5)}=q_{3}+q_{5} p_{2} \text {, } \\
& \Omega_{(3,6)}=q_{3}+q_{6} p_{2}, \Omega_{(3,7)}=\Omega_{(3,8)}=\Omega_{(3,9)}=0 \text {, } \\
& \Omega_{(3,10)}=\Omega_{(3,11)}=\Omega_{(3,12)}=0 \text {, } \\
& \Omega_{(3,13)}=-q_{7} a_{2} h_{1}(x(t-\tau(t))) \text {, } \\
& \Omega_{(3,14)}=\Omega_{(3,15)}=\Omega_{(3,16)}=0 \text {, } \\
& \Omega_{(3,17)}=\Omega_{(3,18)}=\Omega_{(3,19)}=0 \text {, } \\
& \Omega_{(3,20)}=\Omega_{(3,21)}=\Omega_{(3,22)}=0 \text {, } \\
& \Omega_{(3,23)}=\Omega_{(3,24)}=\Omega_{(3,25)}=0, \Omega_{(4,4)}=-2 q_{4} p_{1} \text {, } \\
& \Omega_{(4,5)}=q_{4}-q_{5} p_{1}, \Omega_{(4,6)}=q_{4}-q_{6} p_{1} \text {, } \\
& \Omega_{(4,7)}=\Omega_{(4,8)}=\Omega_{(4,9)}=0 \text {, } \\
& \Omega_{(4,10)}=\Omega_{(4,11)}=\Omega_{(4,12)}=0, \Omega_{(4,13)}=-q_{7} a_{2}, \\
& \Omega_{(4,23)}=\Omega_{(4,24)}=\Omega_{(4,25)}=0 \text {, } \\
& \Omega_{(5,5)}=2 q_{5}-k_{5} e^{-2 \alpha \gamma \tau_{2}}+k_{5} \gamma \tau_{d}, \Omega_{(5,6)}=q_{5}+q_{6} \text {, } \\
& \Omega_{(5,7)}=\Omega_{(5,8)}=\Omega_{(5,9)}=0 \text {, } \\
& \Omega_{(5,10)}=\Omega_{(5,11)}=\Omega_{(5,12)}=0 \text {, } \\
& \Omega_{(5,13)}=\Omega_{(5,14)}=\Omega_{(5,15)}=\Omega_{(5,16)}=0 \text {, } \\
& \Omega_{(5,17)}=\Omega_{(5,18)}=\Omega_{(5,19)}=0 \text {, } \\
& \Omega_{(5,20)}=\Omega_{(5,21)}=\Omega_{(5,22)}=0 \text {, } \\
& \Omega_{(5,23)}=\Omega_{(5,24)}=\Omega_{(5,25)}=0, \Omega_{(6,6)}=2 q_{6} \text {, } \\
& \Omega_{(6,7)}=\Omega_{(6,8)}=\Omega_{(6,9)}=0 \text {, } \\
& \Omega_{(6,10)}=\Omega_{(6,11)}=\Omega_{(6,12)}=0 \text {, } \\
& \Omega_{(6,13)}=\Omega_{(6,14)}=\Omega_{(6,15)}=\Omega_{(6,16)}=0 \text {, } \\
& \Omega_{(6,17)}=\Omega_{(6,18)}=\Omega_{(6,19)}=0 \text {, } \\
& \Omega_{(6,20)}=\Omega_{(6,21)}=\Omega_{(6,22)}=0 \text {, } \\
& \Omega_{(6,23)}=\Omega_{(6,24)}=\Omega_{(6,25)}=0 \text {, } \\
& \Omega_{(7,7)}=-\left(k_{2}+w_{3}\right) e^{-2 \alpha \tau_{2}}, \Omega_{(7,8)}=\Omega_{(7,9)}=0 \text {, } \\
& \Omega_{(7,10)}=\Omega_{(7,11)}=\Omega_{(7,12)}=0 \text {, } \\
& \Omega_{(7,13)}=\Omega_{(7,14)}=\Omega_{(7,15)}=\Omega_{(7,16)}=0 \text {, } \\
& \Omega_{(7,17)}=\Omega_{(7,18)}=\Omega_{(7,19)}=0 \text {, } \\
& \Omega_{(7,20)}=\Omega_{(7,21)}=\Omega_{(7,22)}=0 \text {, } \\
& \Omega_{(7,23)}=\Omega_{(7,24)}=\Omega_{(7,25)}=0, \Omega_{(8,8)}=-k_{6} e^{-2 \alpha \tau_{2}}, \\
& \Omega_{(8,9)}=\Omega_{(8,10)}=\Omega_{(8,11)}=0 \text {, } \\
& \Omega_{(8,12)}=\Omega_{(8,13)}=\Omega_{(8,14)}=0 \text {, } \\
& \Omega_{(8,15)}=\Omega_{(8,16)}=\Omega_{(8,17)}=0 \text {, } \\
& \Omega_{(8,18)}=\Omega_{(8,19)}=\Omega_{(8,20)}=0 \text {, } \\
& \Omega_{(8,21)}=\Omega_{(8,22)}=\Omega_{(8,23)}=\Omega_{(8,24)}=\Omega_{(8,25)}=0 \text {, } \\
& \Omega_{(9,9)}=-\left(k_{4}+w_{4}\right) e^{-2 \alpha \gamma \tau_{2}} \text {, } \\
& \Omega_{(9,10)}=\Omega_{(9,11)}=\Omega_{(9,12)}=0 \text {, } \\
& \Omega_{(9,13)}=\Omega_{(9,14)}=\Omega_{(9,15)}=0 \text {, } \\
& \Omega_{(9,16)}=\Omega_{(9,17)}=\Omega_{(9,18)}=0 \text {, } \\
& \Omega_{(9,19)}=\Omega_{(9,20)}=\Omega_{(9,21)}=0 \text {, } \\
& \Omega_{(9,22)}=\Omega_{(9,23)}=\Omega_{(9,24)}=\Omega_{(9,25)}=0 \text {, } \\
& \Omega_{(10,10)}=-k_{7} e^{-2 \alpha \gamma \tau_{2}} \text {, } \\
& \Omega_{(10,11)}=\Omega_{(10,12)}=\Omega_{(10,13)}=0 \text {, }
\end{aligned}
$$




$$
\begin{aligned}
& \Omega_{(10,14)}=\Omega_{(10,15)}=\Omega_{(10,16)}=0 \text {, } \\
& \Omega_{(10,17)}=\Omega_{(10,18)}=\Omega_{(10,19)}=0 \text {, } \\
& \Omega_{(10,20)}=\Omega_{(10,21)}=\Omega_{(10,22)}=0 \text {, } \\
& \Omega_{(10,23)}=\Omega_{(10,24)}=\Omega_{(10,25)}=0 \text {, } \\
& \Omega_{(11,11)}=-k_{8} e^{-2 \alpha \sigma_{2}}+k_{8} \sigma_{d}-k_{10}, \Omega_{(11,12)}=0 \text {, } \\
& \Omega_{(11,13)}=q_{7} b(t), \Omega_{(11,14)}=\Omega_{(11,15)}=\Omega_{(11,16)}=0 \text {, } \\
& \Omega_{(11,17)}=\Omega_{(11,18)}=\Omega_{(11,19)}=0 \text {, } \\
& \Omega_{(11,20)}=\Omega_{(11,21)}=\Omega_{(11,22)}=0 \text {, } \\
& \Omega_{(11,23)}=\Omega_{(11,24)}=\Omega_{(11,25)}=0 \text {, } \\
& \Omega_{(12,12)}=-k_{9} e^{-2 \alpha \sigma_{2}} \text {, } \\
& \Omega_{(12,13)}=\Omega_{(12,14)}=\Omega_{(12,15)}=\Omega_{(12,16)}=0 \text {, } \\
& \Omega_{(12,17)}=\Omega_{(12,18)}=\Omega_{(12,19)}=0 \text {, } \\
& \Omega_{(12,20)}=\Omega_{(12,21)}=\Omega_{(12,22)}=\Omega_{(12,23)}=0 \text {, } \\
& \Omega_{(12,24)}=\Omega_{(12,25)}=0, \Omega_{(13,13)}=-2 q_{7} \text {, } \\
& \Omega_{(13,14)}=\Omega_{(13,15)}=\Omega_{(13,16)}=0 \text {, } \\
& \Omega_{(13,17)}=\Omega_{(13,18)}=\Omega_{(13,19)}=0 \text {, } \\
& \Omega_{(13,20)}=\Omega_{(13,21)}=\Omega_{(13,22)}=0 \text {, } \\
& \Omega_{(13,23)}=\Omega_{(13,24)}=0, \Omega_{(13,25)}=q_{7} \text {, } \\
& \Omega_{(14,14)}=\left(w_{3}-w_{1}\right) e^{-2 \alpha \tau_{1}}, \Omega_{(14,15)}=\Omega_{(14,16)}=0 \text {, } \\
& \Omega_{(14,17)}=\Omega_{(14,18)}=\Omega_{(14,19)}=\Omega_{(14,20)}=\Omega_{(14,21)}=0 \text {, } \\
& \Omega_{(14,22)}=\Omega_{(14,23)}=\Omega_{(14,24)}=\Omega_{(14,25)}=0 \text {, } \\
& \Omega_{(15,15)}=\left(w_{4}-w_{2}\right) e^{-2 \alpha \gamma \tau_{1}}, \Omega_{(15,16)}=0 \text {, } \\
& \Omega_{(15,17)}=\Omega_{(15,18)}=\Omega_{(15,19)}=\Omega_{(15,20)}=0 \text {, } \\
& \Omega_{(15,21)}=\Omega_{(15,22)}=\Omega_{(15,23)}=\Omega_{(15,24)}=\Omega_{(15,25)}=0 \text {, } \\
& \Omega_{(16,16)}=-w_{5} e^{-2 \alpha \tau_{1}} \text {, } \\
& \Omega_{(16,17)}=\Omega_{(16,18)}=\Omega_{(16,19)}=\Omega_{(16,20)}=\Omega_{(16,21)}=0 \text {, } \\
& \Omega_{(16,22)}=\Omega_{(16,23)}=\Omega_{(16,24)}=\Omega_{(16,25)}=0 \text {, } \\
& \Omega_{(17,17)}=-w_{6} e^{-2 \alpha \gamma \tau_{1}} \text {, } \\
& \Omega_{(17,18)}=\Omega_{(17,19)}=\Omega_{(17,20)}=\Omega_{(17,21)}=0 \text {, } \\
& \Omega_{(17,22)}=\Omega_{(17,23)}=\Omega_{(17,24)}=\Omega_{(17,25)}=0 \text {, } \\
& \Omega_{(18,18)}=-w_{7} e^{-2 \alpha \tau_{2}} \text {, } \\
& \Omega_{(18,19)}=\Omega_{(18,20)}=\Omega_{(18,21)}=\Omega_{(18,22)}=0 \text {, } \\
& \Omega_{(18,23)}=\Omega_{(18,24)}=\Omega_{(18,25)}=0 \text {, } \\
& \Omega_{(19,19)}=-w_{7} e^{-2 \alpha \tau_{2}} \text {, } \\
& \Omega_{(19,20)}=\Omega_{(19,21)}=\Omega_{(19,22)}=0 \text {, }
\end{aligned}
$$

$$
\begin{aligned}
& \Omega_{(19,23)}=\Omega_{(19,24)}=\Omega_{(19,25)}=0, \\
& \Omega_{(20,20)}=-w_{8} e^{-2 \alpha \gamma \tau_{2}}, \Omega_{(20,21)}=\Omega_{(20,22)}=0, \\
& \Omega_{(20,23)}=\Omega_{(20,24)}=\Omega_{(20,25)}=0, \\
& \Omega_{(21,21)}=-w_{8} e^{-2 \alpha \gamma \tau_{2}}, \Omega_{(21,22)}=0, \\
& \Omega_{(21,23)}=\Omega_{(21,24)}=\Omega_{(21,25)}=0, \\
& \Omega_{(22,22)}=-w_{9} e^{-2 \alpha \sigma_{1}}, \\
& \Omega_{(22,23)}=\Omega_{(22,24)}=\Omega_{(22,25)}=0, \\
& \Omega_{(23,23)}=-w_{10} e^{-2 \alpha \sigma_{2}}, \Omega_{(23,24)}=\Omega_{(23,25)}=0, \\
& \Omega_{(24,24)}=-w_{10} e^{-2 \alpha \sigma_{2}}, \Omega_{(24,25)}=0, \\
& \Omega_{(25,25)}=-w e^{-2 \alpha p_{2}}, \sigma_{1}, \sigma_{2}, \sigma_{d}, \tau_{1}, \tau_{2}, \tau_{d}, p_{1}, p_{2}, \alpha \\
& \text { and } \gamma \text { are positive some real constants. }
\end{aligned}
$$$$
\text { result. }
$$

We now state the exponentially stability

Theorem. If there exist positive real constants $w, k_{i}, w_{i}, \quad(i=1,2, \ldots, 10)$, such that the following matrix inequality

$$
\Sigma=\left[\Omega_{(i, j)}\right]_{25 \times 25}<0
$$

holds, then zero solution of NIDE (1.2) is exponentially stable with a decay rate $\alpha$.

Proof. We define Lyapunov-Krasovskii functional candidate for NIDE (1.8) of the form

$$
V\left(t, x_{t}\right)=\sum_{i=1}^{5} V_{i}\left(t, x_{t}\right) \text {, }
$$

where

$$
\begin{aligned}
V_{1}\left(t, x_{t}\right)= & k_{1} D^{2}(t) \\
V_{2}\left(t, x_{t}\right)= & k_{2} \int_{t-\tau_{2}}^{t} e^{2 \alpha(s-t)} x^{2}(s) d s+k_{3} \int_{t-\tau(t)}^{t} e^{2 \alpha(s-t)} x^{2}(s) d s \\
& +k_{4} \int_{t-\gamma \tau_{2}}^{t} e^{2 \alpha(s-t)} x^{2}(s) d s \\
& +k_{5} \int_{t-\gamma \tau(t)}^{t} e^{2 \alpha(s-t)} x^{2}(s) d s \\
& +w_{1} \int_{t-\tau_{1}}^{t} e^{2 \alpha(s-t)} x^{2}(s) d s
\end{aligned}
$$




$$
\begin{aligned}
& +w_{2} \int_{t-\gamma \tau_{1}}^{t} e^{2 \alpha(s-t)} x^{2}(s) d s \\
& +w_{3} \int_{t-\tau_{2}}^{t-\tau_{1}} e^{2 \alpha(s-t)} x^{2}(s) d s \\
& +w_{4} \int_{t-\gamma \tau_{2}}^{t-\gamma \tau_{1}} e^{2 \alpha(s-t)} x^{2}(s) d s, \\
& V_{3}\left(t, x_{t}\right)=k_{6} \tau_{2} \int_{-\tau_{2}}^{0} \int_{t+s}^{t} e^{2 \alpha(\theta-t)} x^{2}(\theta) d \theta d s \\
& +k_{7} \gamma \tau_{2} \int_{-\gamma \tau_{2}}^{0} \int_{t+s}^{t} e^{2 \alpha(\theta-t)} x^{2}(\theta) d \theta d s \\
& +w_{5} \tau_{1} \int_{-\tau_{1}}^{0} \int_{t+s}^{t} e^{2 \alpha(\theta-t)} x^{2}(\theta) d \theta d s \\
& +w_{6} \gamma \tau_{1} \int_{-\gamma \tau_{1}}^{0} \int_{t+s}^{t} e^{2 \alpha(\theta-t)} x^{2}(\theta) d \theta d s \\
& +w_{7}\left(\tau_{2}-\tau_{1}\right) \int_{-\tau_{2}}^{-\tau_{1}} \int_{t+s}^{t} e^{2 \alpha(\theta-t)} x^{2}(\theta) d \theta d s \\
& +w_{8} \gamma\left(\tau_{2}-\tau_{1}\right) \int_{-\gamma \tau_{2}}^{-\gamma \tau_{1}} \int_{t+s}^{t} e^{2 \alpha(\theta-t)} x^{2}(\theta) d \theta d s, \\
& V_{4}\left(t, x_{t}\right)=k_{8} \int_{t-\sigma(t)}^{t} e^{2 \alpha(s-t)} \tanh ^{2} x(s) d s \\
& +k_{9} \sigma_{2} \int_{-\sigma_{2}}^{0} \int_{t+s}^{t} e^{2 \alpha(\theta-t)} \tanh ^{2} x(\theta) d \theta d s \\
& +w_{9} \sigma_{1} \int_{-\sigma_{1}}^{0} \int_{t+s}^{t} e^{2 \alpha(\theta-t)} \tanh ^{2} x(\theta) d \theta d s \\
& +w_{10}\left(\sigma_{2}-\sigma_{1}\right) \\
& \times \int_{-\sigma_{2}}^{-\sigma_{1}} \int_{t+s}^{t} e^{2 \alpha(\theta-t)} \tanh ^{2} x(\theta) d \theta d s, \\
& V_{5}\left(t, x_{t}\right)=w p_{2} \int_{-p_{2}}^{0} \int_{t+s}^{t} e^{2 \alpha(\theta-t)} x^{2}(\theta) d \theta d s \\
& \dot{V}\left(t, x_{t}\right)=\sum_{i=1}^{5} \dot{V}_{i}\left(t, x_{t}\right) \\
& \dot{V}_{1}\left(t, x_{t}\right)=2 k_{1} D(t) \dot{D}(t) \\
& =2 k_{1} D(t)\left[-\left(a_{1}-r_{1}-r_{2}\right) h(x(t))\right. \\
& -\left(a_{2}+r_{1}\right) h(x(t-\tau(t)) \\
& -\left(a_{2}+r_{1}\right) \int_{t-\tau(t)}^{t}(h(x(s)))^{\prime} d s \\
& -r_{2} h(x(t-\gamma \tau(t))) \\
& -r_{2} \int_{t-\gamma \tau(t)}^{t}(h(x(s)))^{\prime} d s \\
& +b(t) \tanh x(t-\sigma(t)) \\
& \left.+\int_{t-p(t)}^{t} C(t, s) x(s) d s\right] \\
& +2 q_{1} D(t)\left[-D(t)+p_{1} x(t)\right. \\
& +p_{2} x(t-\tau(t))+x(t-\gamma \tau(t)) \\
& \left.+\int_{t-\gamma \tau(t)}^{t} \dot{x}(s) d s-p_{1} \int_{t-\tau(t)}^{t} \dot{x}(s) d s\right] \\
& +2 q_{2} x(t)\left[-D(t)+p_{1} x(t)\right. \\
& +p_{2} x(t-\tau(t))+x(t-\gamma \tau(t)) \\
& \left.+\int_{t-\gamma \tau(t)}^{t} \dot{x}(s) d s-p_{1} \int_{t-\tau(t)}^{t} \dot{x}(s) d s\right] \\
& +2 q_{3} x(t-\tau(t))\left[-D(t)+p_{1} x(t)\right.
\end{aligned}
$$




$$
\begin{aligned}
& +p_{2} x(t-\tau(t))+x(t-\gamma \tau(t)) \\
& \left.+\int_{t-\gamma \tau(t)}^{t} \dot{x}(s) d s-p_{1} \int_{t-\tau(t)}^{t} \dot{x}(s) d s\right] \\
& +2 q_{4} \int_{t-\tau(t)}^{t} \dot{x}(s) d s\left[-D(t)+p_{1} x(t)\right. \\
& +p_{2} x(t-\tau(t))+x(t-\gamma \tau(t)) \\
& \left.+\int_{t-\gamma \tau(t)}^{t} \dot{x}(s) d s-p_{1} \int_{t-\tau(t)}^{t} \dot{x}(s) d s\right] \\
& +2 q_{5} x(t-\gamma \tau(t))\left[-D(t)+p_{1} x(t)+p_{2} x(t-\tau(t))\right. \\
& \left.+x(t-\gamma \tau(t))+\int_{t-\gamma \tau(t)}^{t} \dot{x}(s) d s-p_{1} \int_{t-\tau(t)}^{t} \dot{x}(s) d s\right] \\
& +2 q_{6} \int_{t-\gamma \tau(t)}^{t} \dot{x}(s) d s\left[-D(t)+p_{1} x(t)+p_{2} x(t-\tau(t))\right. \\
& \left.+x(t-\gamma \tau(t))+\int_{t-\gamma \tau(t)}^{t} \dot{x}(s) d s-p_{1} \int_{t-\tau(t)}^{t} \dot{x}(s) d s\right] \\
& +2 q_{7} \dot{D}(t)\left[-\dot{D}(t)-a_{1} h(x(t))-a_{2} h(x(t-\tau(t))\right. \\
& -a_{2} \int_{t-\tau(t)}^{t}(h(x(s)))^{\prime} d s+b(t) \tanh x(t-\sigma(t)) \\
& \left.+\int_{t-p(t)}^{t} C(t, s) x(s) d s\right]+2 \alpha k_{1} D^{2}(t)-2 \alpha V_{1}(t) .
\end{aligned}
$$

Hence, in view of the assumptions of the given theorem and the condition $h^{\prime}(x) \geq 1, x \neq 0$, we can derive that

$$
\begin{aligned}
\dot{V}_{1}\left(t, x_{t}\right) \leq & 2 k_{1} D(t)\left[-\left(a_{1}-r_{1}-r_{2}\right) h(x(t))\right. \\
& -\left(a_{2}+r_{1}\right) h_{1}(x(t-\tau(t)) x(t-\tau(t)) \\
& -\left(a_{2}+r_{1}\right) \int_{t-\tau(t)}^{t} \dot{x}(s) d s \\
& -r_{2} h_{1}(x(t-\gamma \tau(t))) x(t-\gamma \tau(t)) \\
& -r_{2} \int_{t-\gamma \tau(t)}^{t} \dot{x}(s) d s \\
& +b(t) \tanh x(t-\sigma(t))
\end{aligned}
$$

$$
\begin{aligned}
& \left.+\int_{t-p(t)}^{t} C(t, s) x(s) d s\right] \\
& +2 q_{1} D(t)\left[-D(t)+p_{1} x(t)\right. \\
& +p_{2} x(t-\tau(t))+x(t-\gamma \tau(t)) \\
& \left.+\int_{t-\gamma \tau(t)}^{t} \dot{x}(s) d s-p_{1} \int_{t-\tau(t)}^{t} \dot{x}(s) d s\right] \\
& +2 q_{2} x(t)\left[-D(t)+p_{1} x(t)\right. \\
& +p_{2} x(t-\tau(t))+x(t-\gamma \tau(t)) \\
& \left.+\int_{t-\gamma \tau(t)}^{t} \dot{x}(s) d s-p_{1} \int_{t-\tau(t)}^{t} \dot{x}(s) d s\right] \\
& +2 q_{3} x(t-\tau(t))\left[-D(t)+p_{1} x(t)+p_{2} x(t-\tau(t))\right. \\
& \left.+x(t-\gamma \tau(t))+\int_{t-\gamma \tau(t)}^{t} \dot{x}(s) d s-p_{1} \int_{t-\tau(t)}^{t} \dot{x}(s) d s\right] \\
& +2 q_{4} \int_{t=\tau(t)}^{t} \dot{x}(s) d s\left[-D(t)+p_{1} x(t)+p_{2} x(t-\tau(t))\right. \\
& \left.+x(t-\gamma \tau(t))+\int_{t-\gamma \tau(t)}^{t} \dot{x}(s) d s-p_{1} \int_{t-\tau(t)}^{t} \dot{x}(s) d s\right] \\
& +2 q_{5} x(t-\gamma \tau(t))\left[-D(t)+p_{1} x(t)+p_{2} x(t-\tau(t))\right. \\
& \left.+x(t-\gamma \tau(t))+\int_{t-\gamma \tau(t)}^{t} \dot{x}(s) d s-p_{1} \int_{t-\tau(t)}^{t} \dot{x}(s) d s\right] \\
& +2 q_{6} \int_{t-\gamma \tau(t)}^{t} \dot{x}(s) d s\left[-D(t)+p_{1} x(t)+p_{2} x(t-\tau(t))\right. \\
& \left.+x(t-\gamma \tau(t))+\int_{t-\gamma \tau(t)}^{t} \dot{x}(s) d s-p_{1} \int_{t-\tau(t)}^{t} \dot{x}(s) d s\right] \\
& +2 q_{7} \dot{D}(t)\left[-\dot{D}(t)-a_{1} h(x(t))-a_{2} h(x(t-\tau(t))\right. \\
& -a_{2} \int_{t-\tau(t)}^{t}(h(x(s)))^{\prime} d s+b(t) \tanh x(t-\sigma(t)) \\
& \left.+\int_{t-p(t)}^{t} C(t, s) x(s) d s\right]+2 \alpha k_{1} D^{2}(t)-2 \alpha V_{1}(t) \\
& \dot{V}_{2}\left(t, x_{t}\right)=\left(k_{2}+k_{3}+k_{4}+k_{5}+w_{1}+w_{2}\right) x^{2}(t) \\
& -\left(k_{2}+w_{3}\right) e^{-2 \alpha \tau_{2}} x^{2}\left(t-\tau_{2}\right)
\end{aligned}
$$




$$
\begin{gathered}
-k_{3}(1-\dot{\tau}(t)) e^{-2 \alpha \tau(t)} x^{2}(t-\tau(t)) \\
-\left(k_{4}+w_{4}\right) e^{-2 \alpha \gamma \tau_{2}} x^{2}\left(t-\gamma \tau_{2}\right) \\
-k_{5}(1-\gamma \dot{\tau}(t)) e^{-2 \alpha \gamma \tau(t)} x^{2}(t-\gamma \tau(t)) \\
+\left(w_{3}-w_{1}\right) e^{-2 \alpha \tau_{1}} x^{2}\left(t-\tau_{1}\right) \\
+\left(w_{4}-w_{2}\right) e^{-2 \alpha \tau_{1}} x^{2}\left(t-\gamma \tau_{1}\right)-2 \alpha V_{2}(t) \\
\leq\left(k_{2}+k_{3}+k_{4}+k_{5}+w_{1}+w_{2}\right) x^{2}(t) \\
-\left(k_{2}+w_{3}\right) e^{-2 \alpha \tau_{2}} x^{2}\left(t-\tau_{2}\right)-k_{3} e^{-2 \alpha \tau_{2}} x^{2}(t-\tau(t)) \\
+k_{3} \tau_{d} x^{2}(t-\tau(t))-\left(k_{4}+w_{4}\right) e^{-2 \alpha \gamma \tau_{2}} x^{2}\left(t-\gamma \tau_{2}\right) \\
-k_{5} e^{-2 \alpha \gamma \tau_{2}} x^{2}(t-\gamma \tau(t))+k_{5} \gamma \tau_{d} x^{2}(t-\gamma \tau(t)) \\
+\left(w_{3}-w_{1}\right) e^{-2 \alpha \tau_{1}} x^{2}\left(t-\tau_{1}\right) \\
+\left(w_{4}-w_{2}\right) e^{-2 \alpha \tau_{1}} x^{2}\left(t-\gamma \tau_{1}\right)-2 \alpha V_{2}(t) .
\end{gathered}
$$

Obviously, for any scalars $s \in\left[t-\tau_{2}, t\right]$ and $s \in\left[t-\gamma \tau_{2}, t\right]$, we can get $e^{-2 \alpha \tau_{2}} \leq e^{2 \alpha(s-t)} \leq 1$ and $e^{-2 \alpha \gamma \tau_{2}} \leq e^{2 \alpha(s-t)} \leq 1$, respectively. In view of Lemma 1.1 and Lemma 1.2, the given assumptions, from $V_{3}\left(t, x_{t}\right)$ and $\operatorname{NIDE}(1.2)$, it follows that

$$
\begin{aligned}
\dot{V}_{3}\left(t, x_{t}\right)= & k_{6} \tau_{2} \int_{-\tau_{2}}^{0} x^{2}(t) d s-k_{6} \tau_{2} \int_{-\tau_{2}}^{0} e^{2 s \alpha} x^{2}(t+s) d s \\
+ & k_{7} \gamma \tau_{2} \int_{-\gamma \tau_{2}}^{0} x^{2}(t) d s \\
& -k_{7} \gamma \tau_{2} \int_{-\gamma \tau_{2}}^{0} e^{2 s \alpha} x^{2}(t+s) d s \\
& +w_{5} \tau_{1} \int_{-\tau_{1}}^{0} x^{2}(t) d s \\
& -w_{5} \tau_{1} \int_{-\tau_{1}}^{0} e^{2 s \alpha} x^{2}(t+s) d s \\
& +w_{6} \gamma \tau_{1} \int_{-\gamma \tau_{1}}^{0} x^{2}(t) d s \\
& -w_{6} \gamma \tau_{1} \int_{-\gamma \tau_{1}}^{0} e^{2 s \alpha} x^{2}(t+s) d s
\end{aligned}
$$

$$
\begin{gathered}
+w_{7}\left(\tau_{2}-\tau_{1}\right) \int_{-\tau_{2}}^{-\tau_{1}} x^{2}(t) d s \\
-w_{7}\left(\tau_{2}-\tau_{1}\right) \int_{-\tau_{2}}^{-\tau_{1}} e^{2 s \alpha} x^{2}(t+s) d s \\
\left.+w_{8}\left(\gamma \tau_{2}-\gamma \tau_{1}\right) \int_{-\gamma \tau_{2}}^{-\lambda \tau_{1}} e^{2 s \alpha} x^{2}(t+s) d s-2 \alpha \tau_{2}-\gamma \tau_{1}\right) \int_{-\gamma \tau_{2}}^{-\gamma \tau_{1}} x^{2}(t) d s \\
+k_{6}\left(\tau_{2}\right)^{2} x^{2}(t)-k_{6} e^{-2 \alpha \tau_{2}}\left(\int_{t-\tau_{2}}^{t} x(s) d s\right)^{2} \\
+w_{8} e^{-2 \gamma \alpha \tau_{2}}\left(\int_{t-\gamma \tau_{2}}^{t-\gamma \tau(t)} x(s) d s\right)^{2}-2 \alpha \tau_{2}\left(\int_{t-\gamma \tau(t)}^{t-\gamma \tau_{1}} x(s) d s\right)^{2} v_{7}(t) . \\
+w_{7}\left(\gamma \tau_{2}\right)^{2} x^{2}(t)-k_{7} e^{-2 \alpha \gamma \tau_{2}}\left(\int_{t-\gamma \tau_{2}}^{t} x(s) d s\right)^{2} \\
+w_{6}\left(\gamma \tau_{1}\right)^{2} x^{2}(t)-w_{6} e^{-2 \alpha \gamma \tau_{1}}\left(\int_{t-\gamma \tau_{1}}^{t} x(s) d s\right)^{2}(t)-w_{8}\left(\gamma \tau_{2}-\gamma \tau_{1}\right)^{2} x^{2}(t) \\
+w_{5}\left(\tau_{1}\right)^{2} x^{2}(t)-w_{5} e^{-2 \alpha \tau_{1}}\left(\int_{t-\tau_{1}}^{t} x(s) d s\right)^{2} \mathrm{~b}
\end{gathered}
$$

Similarly, by using Lemma 1.1, Lemma 1.2, the inequality $\quad \tanh ^{2} x(t) \leq x^{2}(t), \quad V_{4}\left(t, x_{t}\right)$ and NIDE (1.2), we have

$$
\dot{V}_{4}\left(t, x_{t}\right)=k_{8} \tanh ^{2} x(t)
$$




$$
\begin{aligned}
& -k_{8}(1-\dot{\sigma}(t)) e^{-2 \alpha \sigma(t)} \tanh ^{2} x(t-\sigma(t)) \\
& +k_{8} \sigma_{2} \int_{-\sigma_{2}}^{0} \tanh ^{2} x(t) d s \\
& -k_{9} \sigma_{2} \int_{-\sigma_{2}}^{0} e^{2 \alpha s} \tanh ^{2} x(t+s) d s
\end{aligned}
$$

$$
\leq w p_{2}^{2} x^{2}(t)-w p_{2} e^{-2 \alpha p_{2}} \int_{t-p_{2}}^{t} x^{2}(s) d s
$$$$
-2 \alpha V_{5}(t)
$$

$$
\leq w p_{2}^{2} x^{2}(t)-w e^{-2 \alpha p_{2}}\left(\int_{t-p_{2}}^{t} x(s) d s\right)^{2}
$$

$+w_{9} \sigma_{1} \int_{-\sigma_{1}}^{0} \tanh ^{2} x(t) d s$

$$
-2 \alpha V_{5}(t)
$$

$-w_{9} \sigma_{1} \int_{-\sigma_{1}}^{0} e^{2 \alpha s} \tanh ^{2} x(t+s) d s$

$$
\begin{aligned}
& \leq w p_{2}^{2} x^{2}(t)-w e^{-2 \alpha p_{2}}\left(\int_{t-p(t)}^{t} x(s) d s\right)^{2}-2 \alpha V_{5}(t) \\
& \leq w p_{2}^{2} x^{2}(t)-w e^{-2 \alpha p_{2}}\left(\int_{t-p(t)}^{t} C(t, s) x(s) d s\right)^{2}
\end{aligned}
$$

$-w_{10}\left(\sigma_{2}-\sigma_{1}\right) \int_{-\sigma_{2}}^{-\sigma_{1}} e^{2 \alpha s} \tanh ^{2} x(t+s) d s-2 \alpha V_{4}(t)$

$-2 \alpha V_{5}(t)$.

$\leq k_{8} \tanh ^{2} x(t)-k_{8} e^{-2 \alpha \sigma_{2}} \tanh ^{2} x(t-\sigma(t))$

On gathering the above discussion in (2.3), we arrive at

$$
\begin{aligned}
& -k_{8} \sigma_{d} \tanh ^{2} x(t-\sigma(t)) \\
& \dot{V}\left(t, x_{t}\right)+2 \alpha V\left(t, x_{t}\right) \\
& \leq 2 k_{1} D(t)\left[-\left(a_{1}-r_{1}-r_{2}\right) h(x(t))\right. \\
& +k_{9} \sigma_{2}{ }^{2} x^{2}(t)-k_{9} e^{-2 \alpha \sigma_{2}}\left(\int_{t-\sigma_{2}}^{t} \tanh x(s) d s\right)^{2} \\
& -\left(a_{2}+r_{1}\right) h_{1}(x(t-\tau(t)) x(t-\tau(t)) \\
& -\left(a_{2}+r_{1}\right) \int_{t-\tau(t)}^{t} \dot{x}(s) d s \\
& +w_{9} \sigma_{1}^{2} x^{2}(t)-w_{9} e^{-2 \alpha \sigma_{1}}\left(\int_{t-\sigma_{1}}^{t} \tanh x(s) d s\right)^{2} \\
& +w_{10}\left(\sigma_{2}-\sigma_{1}\right)^{2} x^{2}(t) \\
& -w_{10} e^{-2 \alpha \sigma_{2}}\left(\int_{t-\sigma(t)}^{t-\tau_{1}} \tanh x(s) d s\right)^{2} \\
& \dot{V}_{5}\left(t, x_{t}\right)=w p_{2} \int_{-p_{2}}^{0}\left[x^{2}(t)-e^{-2 \alpha s} x^{2}(t+s)\right] d s-2 \alpha V_{5}(t) \\
& -r_{2} h_{1}(x(t-\gamma \tau(t))) x(t-\gamma \tau(t))-r_{2} \int_{t-\gamma \tau(t)}^{t} \dot{x}(s) d s \\
& \left.+b(t) \tanh x(t-\sigma(t))+\int_{t-p(t)}^{t} C(t, s) x(s) d s\right] \\
& +2 q_{1} D(t)\left[-D(t)+p_{1} x(t)+p_{2} x(t-\tau(t))\right. \\
& \left.+x(t-\gamma \tau(t))+\int_{t-\gamma \tau(t)}^{t} \dot{x}(s) d s-p_{1} \int_{t-\tau(t)}^{t} \dot{x}(s) d s\right] \\
& +2 q_{2} x(t)\left[-D(t)+p_{1} x(t)+p_{2} x(t-\tau(t))\right. \\
& =w p_{2}^{2} x^{2}(t)-w p_{2} \int_{-p_{2}}^{0} e^{2 \alpha s} x^{2}(t+s) d s \\
& \left.+x(t-\gamma \tau(t))+\int_{t-\gamma \tau(t)}^{t} \dot{x}(s) d s-p_{1} \int_{t-\tau(t)}^{t} \dot{x}(s) d s\right] \\
& -2 \alpha V_{5}(t) \\
& +2 q_{3} x(t-\tau(t))\left[-D(t)+p_{1} x(t)+p_{2} x(t-\tau(t))\right. \\
& \left.+x(t-\gamma \tau(t))+\int_{t-\gamma \tau(t)}^{t} \dot{x}(s) d s-p_{1} \int_{t-\tau(t)}^{t} \dot{x}(s) d s\right]
\end{aligned}
$$




$$
\begin{aligned}
& +2 q_{4} \int_{t-\tau(t)}^{t} \dot{x}(s) d s\left[-D(t)+p_{1} x(t)+p_{2} x(t-\tau(t))\right. \\
& -w_{7} e^{-2 \alpha \tau_{2}}\left(\int_{t-\tau(t)}^{t-\tau_{1}} x(s) d s\right)^{2}-w_{7} e^{-2 \alpha \tau_{2}}\left(\int_{t-\tau_{2}}^{t-\tau(t)} x(s) d s\right)^{2} \\
& \left.+x(t-\gamma \tau(t))+\int_{t-\gamma \tau(t)}^{t} \dot{x}(s) d s-p_{1} \int_{t-\tau(t)}^{t} \dot{x}(s) d s\right] \\
& +2 q_{5} x(t-\gamma \tau(t))\left[-D(t)+p_{1} x(t)+p_{2} x(t-\tau(t))\right. \\
& \left.+x(t-\gamma \tau(t))+\int_{t-\gamma \tau(t)}^{t} \dot{x}(s) d s-p_{1} \int_{t-\tau(t)}^{t} \dot{x}(s) d s\right] \\
& +2 q_{6} \int_{t-\gamma \tau(t)}^{t} \dot{x}(s) d s\left[-D(t)+p_{1} x(t)+p_{2} x(t-\tau(t))\right. \\
& \left.+x(t-\gamma \tau(t))+\int_{t-\gamma \tau(t)}^{t} \dot{x}(s) d s-p_{1} \int_{t-\tau(t)}^{t} \dot{x}(s) d s\right] \\
& +2 q_{7} \dot{D}(t)\left[-\dot{D}(t)-a_{1} h(x(t))-a_{2} h(x(t-\tau(t))\right. \\
& -a_{2} \int_{t-\tau(t)}^{t}(h(x(s)))^{\prime} d s+b(t) \tanh x(t-\sigma(t)) \\
& \left.+\int_{t-p(t)}^{t} C(t, s) x(s) d s\right]+2 \alpha k_{1} D^{2}(t)-2 \alpha V_{1}(t) \\
& +\left(k_{2}+k_{3}+k_{4}+k_{5}+w_{1}+w_{2}\right) x^{2}(t) \\
& -\left(k_{2}+w_{3}\right) e^{-2 \alpha \tau_{2}} x^{2}\left(t-\tau_{2}\right) \\
& -k_{3} e^{-2 \alpha \tau_{2}} x^{2}(t-\tau(t))+k_{3} \tau_{d} x^{2}(t-\tau(t)) \\
& -\left(k_{4}+w_{4}\right) e^{-2 \alpha \gamma \tau_{2}} x^{2}\left(t-\gamma \tau_{2}\right) \\
& -k_{5} e^{-2 \alpha \gamma \tau_{2}} x^{2}(t-\gamma \tau(t))+k_{5} \gamma \tau_{d} x^{2}(t-\gamma \tau(t)) \\
& +\left(w_{3}-w_{1}\right) e^{-2 \alpha \tau_{1}} x^{2}\left(t-\tau_{1}\right) \\
& +\left(w_{4}-w_{2}\right) e^{-2 \alpha \tau_{1}} x^{2}\left(t-\gamma \tau_{1}\right)-2 \alpha V_{2}(t) \\
& \begin{array}{l}
-w_{8} e^{-2 \gamma \alpha \tau_{2}}\left(\int_{t-\gamma \tau(t)}^{t-\gamma \tau_{1}} x(s) d s\right)^{2} \\
-w_{8} e^{-2 \gamma \alpha \tau_{2}}\left(\int_{t-\gamma \tau_{2}}^{t-\gamma \tau(t)} x(s) d s\right)^{2}-2 \alpha V_{3}(t)
\end{array} \\
& +k_{8} \tanh ^{2} x(t)-k_{8} e^{-2 \alpha \sigma_{2}} \tanh ^{2} x(t-\sigma(t)) \\
& -k_{8} \sigma_{d} \tanh ^{2} x(t-\sigma(t)) \\
& +k_{9} \sigma_{2}^{2} x^{2}(t)-k_{9} e^{-2 \alpha \sigma_{2}}\left(\int_{t-\sigma_{2}}^{t} \tanh x(s) d s\right)^{2} \\
& +w_{9} \sigma_{1}^{2} x^{2}(t)-w_{9} e^{-2 \alpha \sigma_{1}}\left(\int_{t-\sigma_{1}}^{t} \tanh x(s) d s\right)^{2} \\
& +w_{10}\left(\sigma_{2}-\sigma_{1}\right)^{2} x^{2}(t) \\
& -w_{10} e^{-2 \alpha \sigma_{2}}\left(\int_{t-\sigma(t)}^{t-\tau_{1}} \tanh x(s) d s\right)^{2} \\
& +k_{10} x^{2}(t)-k_{10} \tanh ^{2} x(t) \\
& -w_{10} e^{-2 \alpha \sigma_{2}}\left(\int_{t-\sigma_{2}}^{t-\sigma(t)} \tanh x(s) d s\right)^{2}-2 \alpha V_{4}(t) \\
& +w p_{2}^{2} x^{2}(t)-w e^{-2 \alpha p_{2}}\left(\int_{t-p(t)}^{t} C(t, s) x(s) d s\right)^{2} \\
& -2 \alpha V_{5}(t) .
\end{aligned}
$$$$
+k_{6}\left(\tau_{2}\right)^{2} x^{2}(t)-k_{6} e^{-2 \alpha \tau_{2}}\left(\int_{t-\tau_{2}}^{t} x(s) d s\right)^{2}
$$$$
+k_{7}\left(\gamma \tau_{2}\right)^{2} x^{2}(t)-k_{7} e^{-2 \alpha \gamma \tau_{2}}\left(\int_{t-\gamma \tau_{2}}^{t} x(s) d s\right)^{2}
$$$$
+w_{5}\left(\tau_{1}\right)^{2} x^{2}(t)-w_{5} e^{-2 \alpha \tau_{1}}\left(\int_{t-\tau_{1}}^{t} x(s) d s\right)^{2}
$$$$
+w_{6}\left(\gamma \tau_{1}\right)^{2} x^{2}(t)-w_{6} e^{-2 \alpha \gamma \tau_{1}}\left(\int_{t-\gamma \tau_{1}}^{t} x(s) d s\right)^{2}
$$$$
+w_{7}\left(\tau_{2}-\tau_{1}\right)^{2} x^{2}(t)-w_{8}\left(\gamma \tau_{2}-\gamma \tau_{1}\right)^{2} x^{2}(t)
$$

Then, it be followed that $\dot{V}\left(t, x_{t}\right)+2 \alpha V\left(t, x_{t}\right) \leq \xi^{T}(t) \sum \xi(t)$, where

$$
\begin{gathered}
\xi^{T}(t)=\left[D(t), x(t), x(t-\tau(t)), \int_{t-\tau(t)}^{t} \dot{x}(s) d s, x(t-\gamma \tau(t)),\right. \\
\int_{t-\gamma \tau(t)}^{t} \dot{x}(s) d s, x\left(t-\tau_{2}\right), \int_{t-\tau_{2}}^{t} x(s) d s, x\left(t-\gamma \tau_{2}\right), \\
\int_{t-\gamma \tau_{2}}^{t} x(s) d s, \tanh x(t-\sigma(t)),
\end{gathered}
$$




$$
\begin{aligned}
& \int_{t-\sigma_{2}}^{t} \tanh x(s) d s, \dot{D}(t), x\left(t-\tau_{1}\right), \\
& x\left(t-\gamma \tau_{1}\right), \int_{t-\tau_{1}}^{t} x(s) d s, \int_{t-\gamma \tau_{1}}^{t} x(s) d s, \\
& \int_{t-\tau(t)}^{t-\tau_{1}} x(s) d s, \int_{t-\tau_{2}}^{t-\tau(t)} x(s) d s, \int_{t-\gamma \tau(t)}^{t-\gamma \tau_{1}} x(s) d s, \\
& \int_{t-\gamma \tau_{2}}^{t-\gamma \tau(t)} x(s) d s, \int_{t-\sigma_{1}}^{t} \tanh x(s) d s, \\
& \left.\int_{t-\sigma_{2}}^{t-\sigma(t)} \tanh x(s) d s, \int_{t-p(t)}^{t} C(t, s) x(s) d s\right]
\end{aligned}
$$

and $\Sigma$ is defined by (2.1). If the condition (2.2) holds, then

$$
\dot{V}\left(t, x_{t}\right)+2 \alpha V\left(t, x_{t}\right) \leq 0, \forall t \in R^{+} .
$$

From (2.4), it is easy to see that

$$
\|x(t, \phi)\| \leq \beta\|\phi\| e^{-\alpha t}, t \in R^{+} .
$$

This means that equation (1.2) is exponentially stable. The proof of the theorem is complete.

\section{Remark}

It is clear that the derivative given by (2.3) is a quadratic form with respect to the variables in (2.4). Then, this derivative can be arranged as a quadratic form. In that case, we can obtain the matrix (2.2).

\section{Conclusion}

The exponential stability of zero solution of a neutral integro-differential equation of first order (NIDE) with discrete and distributed time-varying delays was investigated. A theorem was proved on the exponential stability of the zero solution of the considered NIDE by means of a new defined LyapunovKrasovskii functional. Our result improves and includes some results that can be found in the literature.

\section{References}

Agarwal, R. P.; Grace, S. R. 2000. 'Asymptotic stability of certain neutral differential equations'. Math. Comput. Modelling 31, no. 8-9, 9-15.

Boyd, Stephen; El Ghaoui, Laurent; Feron, Eric; Balakrishnan, Venkataramanan, 1994. 'Linear matrix inequalities in system and control theory'. SIAM Studies in Applied Mathematics, 15. Society for Industrial and Applied Mathematics (SIAM), Philadelphia, PA,

Chen, Huabin; Meng, Xuejing, 2011. 'An improved exponential stability criterion for a class of neutral delayed differential equations'. Appl. Math. Lett. 24, no. 11, 1763-1767.

Chen, Huabin, 2012. 'Some improved criteria on exponential stability of neutral differential equation'. Adv. Difference Equ., 2012:170, 9 pp.

Chatbupapan, Watcharin; Mukdasai, Kanit, 2016. 'New delay-range-dependent exponential stability criteria for certain neutral differential equations with interval discrete and distributed time- varying delays'. Adv. Difference Equ., Paper No. 324, 18 pp.

Deng, Shaojiang; Liao, Xiaofeng; Guo, Songtao, 2009. 'Asymptotic stability analysis of certain neutral differential equations: a descriptor system approach'. Math. Comput. Simulation 79, no. 10, 2981-2993.

El-Morshedy, H. A.; Gopalsamy, K., 2000. 'Nonoscillation, oscillation and convergence of a class of neutral equations'. Lakshmikantham's legacy: a tribute on his 75th birthday. Nonlinear Anal. 40, no. 1-8, Ser. A: Theory Methods, 173-183.

Fridman, E. 2001. 'New Lyapunov-Krasovskii functionals for stability of linear retarded and neutral type systems'. Systems Control Lett. 43, no. 4, 309-319.

Fridman, Emilia, 2002. 'Stability of linear descriptor systems with delay: a Lyapunov- 
based approach'. J. Math. Anal. Appl. 273, no. $1,24-44$.

Gu, Keqin; Kharitonov, Vladimir L.; Chen, Jie, 2003. 'Stability of time-delay systems'. Control Engineering. Birkhäuser Boston, Inc., Boston, MA.

Gözen, Melek; Tunc, Cemil 2017. 'On exponential stability of solutions of neutral differential systems with multiple variable delays'. Electron. J. Math. Anal. Appl. 5, no. 1, $17-31$.

Gözen, Melek; Tunç, Cemil 2017. 'A note on the exponential stability of linear systems with variable retardations'. Appl. Math. Inf. Sci. 11, no. 3, 899-906.

Gözen, Melek; Tunç, Cemil 2018. 'On the exponential stability of a neutral differential equation of first order'. J. Math. Appl. 41, 95107.

Hale, Jack K.; Verduyn Lunel, Sjoerd M. 1993. 'Introduction to functional-differential equations'. Applied Mathematical Sciences, 99. Springer- Verlag, New York.

Hristova, Snezhana; Tunc, Cemil 2019. 'Stability of nonlinear Volterra integroDifferential equations with Caputo fractional derivative and bounded delays'. Electron. J. Differential Equations, Paper No. 30, 11 pp.

Kwon, O. M.; Park, Ju H., 2006. 'Exponential stability of uncertain dynamic systems including state delay'. Appl. Math. Lett. 19, no. 9, 901907.

Kwon, O. M.; Park, Ju H., 2008. 'On improved delay-dependent stability criterion of certain neutral differential equations'. Appl. Math. Comput. 199, no. 1, 385-391.

Keadnarmol, Panuwat; Rojsiraphisal, Thaned, 2014. 'Globally exponential stability of a certain neutral differential equation with time-varying delays'. Adv. Difference Equ., 32, 10 pp.

Liao, Xiaofeng; Liu, Yanbing; Guo, Songtao; Mai, Huanhuan, 2009. 'Asymptotic stability of delayed neural networks: a descriptor system approach'. Commun. Nonlinear Sci. Numer. Simul. 14, no. 7, 3120-3133.

Li, Xiaodi 2009. 'Global exponential stability for a class of neural networks'. Appl. Math. Lett. 22 , no. $8,1235-1239$.

Li, Xiaodi; Fu, Xilin, 2013. 'Effect of leakage time-varying delay on stability of nonlinear differential systems'. J. Franklin Inst. 350, no. $6,1335-1344$.

Nam, P. T.; Phat, V. N. 2009. 'An improved stability criterion for a class of neutral differential equations'. Appl. Math. Lett. 22, no. 1, 31-35.

Park, J. H. 2004. 'Delay-dependent criterion for asymptotic stability of a class of neutral equations'. Appl. Math. Lett. 17, no. 10, 1203 1206.

Park, Ju H.; Kwon, O. M., 2008. 'Stability analysis of certain nonlinear differential equation'. Chaos Solitons Fractals 37, no. 2, $450-453$.

Pinjai, Sirada; Mukdasai, Kanit, 2013. 'New delay-dependent robust exponential stability criteria of LPD neutral systems with mixed time-varying delays and nonlinear perturbations'. J. Appl. Math., Art. ID 268905, $18 \mathrm{pp}$.

Rojsiraphisal, Thaned; Niamsup, Piyapong 2010. 'Exponential stability of certain neutral differential equations'. Appl. Math. Comput. 217 , no. 8, 3875-3880.

Sun, Yuan Gong; Wang, Long 2006. 'Note on asymptotic stability of a class of neutral differential equations'. Appl. Math. Lett. 19, no. 9, 949-953.

Slyn'ko, Vitalii; Tunç, Cemil; 2019. 'Stability of abstract linear switched impulsive differential equations'. Automatica J. IFAC 107, 433-441.

Tunç, Cemil; Altun, Melek 2012. 'On the integrability of solutions of non-autonomous 
differential equations of second order with multiple variable deviating arguments'. J. Comput. Anal. Appl. 14, no. 5, 899-908.

Tunç, Cemil 2013. 'Exponential stability to a neutral differential equation of first order with delay'. Ann. Differential Equations 29, no. 3, 253-256.
Tunç, Cemil; Mohammed, Sizar Abid 2017. 'New results on exponential stability of nonlinear Volterra integro-differential equations with constant time-lag'. Proyecciones 36, no. 4, Tunç, Cemil; Tunç, Osman 2018. 'New results on the stability, integrability and boundedness in Volterra integro-differential equations'. Bull. Comput. Appl. Math. 6, no. 1, 41-58. 